\title{
Lumbosacral Region
}

National Cancer Institute

\section{Source}

National Cancer Institute. Lumbosacral Region. NCI Thesaurus. Code C48824.

The region of the spine in the lower back that consists of the five lumbar vertebrae and the sacrum. 